EDO-EP-29

September, 1999

\title{
Mass Hierarchy and Trapping of Gravity
}

\author{
Ichiro Oda f \\ Edogawa University, 474 Komaki, Nagareyama City, Chiba 270-0198, JAPAN
}

\begin{abstract}
We construct a model consisting of many D3-branes with only positive tension in a five-dimensional anti-de Sitter space-time geometry. It is shown that this type of model naturally realizes not only exponential mass hierarchy between the Planck scale and the electroweak scale but also trapping of the graviton on the D3-branes. It is pointed out that our model may have a flexibility to explain the existence of more than one disparate mass scales, such as the electroweak scale and the GUT scale, on the same D3-brane.
\end{abstract}

\footnotetext{
${ }^{1}$ E-mail address: ioda@edogawa-u.ac.jp.
} 


\section{Introduction}

Understanding the hierarchy problem of the Standard Model in four dimensions is a classic and important problem. The main ideas for this problem have been so far focused on improvement of particle physics sector around the TeV scale. Recently, however, motivated by studies of non-perturbative superstring theory and M-theory, a completely new proposal for the hierarchy problem has been made in gravity sector where the huge Planck scale is reduced all the way to the $\mathrm{TeV}$ scale by assuming the existence of extra dimensions [1, 2, 3].

In particular, Randall and Sundrum have considered a solution for the five-dimensional Einstein equation with the cosmological constant and two 3-branes [3, 4. This solution involves a "red-shift" (or "warp") factor in the metric tensor, which was found to play a critical role in explaining the vast disparity between the Planck scale and the electroweak scale in a natural way [3]. Subsequently, they proposed an alternative scenario for trapping of gravity [4].

Here it is worth summarizing basic setups and aims of the two papers [3, 4] since they have proposed two distinct new scenarios in terms of the same solution as stated above. In both the papers, two 3-branes, in other words, domain walls, are placed at the boundaries of the fifth dimension, which are the fixed points of an orbifold $S^{1} / Z_{2}$, in the five-dimensional

anti-de Sitter space-time [5, 6]. The one 3-brane with positive brane tension is located at the origin of the fifth dimension while the other 3-brane with opposite brane tension to the first 3-brane is located at another fixed point away from the origin at some distance. Note that one has to make such a fine-tuning of brane tensions to obtain the Randall-Sundrum solution for the Einstein equation. (This issue will be argued in some detail around the end of section 2.)

In the first paper [3], our universe is assumed to the 3-brane with negative tension whereas the other 3-brane with positive tension is regarded as hidden universe. If we think that a natural scale on the hidden world is of order the Planck scale, the electroweak scale is generated on our universe from the red-shift factor by selecting rather small size of an extra dimension. Thus, the purpose of the paper is to present a resolution to the hierarchy problem.

On the other hand, in the second paper [4, the model setup is converse compared to that in the first paper [3]. That is, our universe is assumed to the 3-brane with positive tension at the origin whereas the other 3-brane with negative tension is regarded as hidden universe. Moreover, the 3-brane with negative tension is moved to infinity, thus providing an example of a non-compact extra dimension. A remarkable thing here is that even without mass gap between the massless graviton and the continuous Kaluza-Klein spectrum, the fourdimensional Newton's law is reproduced to more than adequate precision on our universe, thereby implying the trapping of gravity on our universe. The reason why the 3-brane with positive tension is taken to be our universe is that the 3-brane with positive tension supplies us with the $\delta$-function potential with a negative coefficient supporting the massless graviton, which will be discussed in section 4 . Of course, in this setup, we cannot solve the hierarchy problem.

From these considerations, it is natural to ask whether one can construct a model with 
the same geometry as in the Randall-Sundrum metric solution such that problems of mass hierarchy and trapping of gravity on our 3-brane universe are simultaneously solved. Indeed, in a recent work [7], Lykken and Randall have explored such a possibility by considering a model including only 3-branes with positive tension. Their setup is similar to that in the second paper [4], but is distinct in that the setup includes more than one positive tension 3brane. But it is unclear at least for the present author that introduction of many 3-branes with positive tension in addition to a single 3-brane with negative tension ("regulator brane") in the setup is consistent with the Einstein equation. One of motivations in this paper is to show explicitly that the above setup is indeed compatible with the Einstein equation. However, to do so, at the outset we need to introduce the same number of negative tension 3-branes as positive tension 3-branes into a model, and after that we have to take a suitable limit in order to move negative tension 3-branes to infinity. Of course, in an extreme case where all the negative tension 3-branes are coincident, our model would become equivalent to that of Lykken and Randall.

The other motivations behind the study at hand are as follows. In this paper, we make use of solutions satisfying the Einstein equation, which were found in our previous work 8 . As stated in the paper, these new solutions describe many domain walls standing along the fifth dimension with topology $S^{1}$ in five-dimensional anti-de Sitter space-time, so they realize many universe cosmology. But these solutions involve the same number of positive tension 3-branes as negative tension 3-branes, thus as a result, the total number of 3-branes are only even. It is quite unfair and against democracy that only even universes can exist in such a model. It will be shown later that we can construct a more plausible model with any number of D3-branes with positive tension by moving negative tension O3-planes to infinity.

Another important motivation of this study is relevant to a recent interesting work by Dienes et al [9]. In their paper, some phenomenological difficulties associated with the RandallSundrum scenario and its extension were pointed out and possible resolutions to these puzzles are speculated. Specially, they have concluded that in the Randall-Sundrum model we cannot simultaneously generate the Planck/electroweak hierarchy (mass hierarchy) and explain gauge coupling unification. This conclusion is physically sensible, of course, as in the original Randall-Sundrum model, there exists only a single red-shift factor depending on a relative distance between the two 3-branes. This red-shift factor operates universally on all mass scales in our universe so it is difficult to generate two mass scales, such as the electroweak scale and the GUT scale, on our universe without recourse to additional mechanisms. One interesting resolution to this puzzle is to consider at least three universe model where one D3-brane is regarded as our universe and the remaining two D3-branes are taken to be hidden universes. As there are two red-shift factors in this model, one could explain the Planck/electroweak hierarchy as well as the existence of the GUT scale on our universe at the same time [10].

The organization of the paper is as follows: in section 2 we review our previous study [8]. Here new solutions for the Einstein equation are presented. Moreover, it is stated in detail about the reason why it is difficult to construct a model with only positive tension branes. In section 3 , based on the solutions reveiwed in section 2 , we construct a model with two positive 
tension 3-branes in a concrete way. The generalization to arbitrary number of positive tension branes is also discussed. In section 4, we investigate mass hierarchy and trapping of gravity along a similar line of arguments to the previous works [4, 7]. The final section is devoted to discussions and future directions of this work.

\section{Review of many domain wall model}

We begin by briefly reviewing a model of Ref. 80. This will enable us to establish our notations and conventions and explain why it is difficult to construct a model consisting of only D3branes with positive tension.

Our starting action is the Einstein-Hilbert action with the cosmological constant in five dimensions plus an action describing many domain walls in four dimensions [8]:

$$
S=\frac{1}{2 \kappa^{2}} \int d^{4} x \int_{0}^{2 L} d z \sqrt{-g}(R-2 \Lambda)+\sum_{i=1}^{n} \int_{z=L_{i}} d^{4} x \sqrt{-g_{i}} \mathcal{L}_{i}
$$

where the cosmological constant $\Lambda$ is taken to a negative number, which implies that the geometry of five-dimensional bulk is anti-de Sitter space-time. The fifth dimension $z$ is assumed to be compact with the length $2 L$, but later regarded to be effectively non-compact by taking the limit $L \rightarrow \infty$. Moreover, $\kappa$ denotes the five-dimensional gravitational constant with a relation $\kappa^{2}=8 \pi G_{N}=\frac{8 \pi}{M_{*}^{3}}$ where $G_{N}$ and $M_{*}$ are the five-dimensional Newton constant and the five-dimensional Planck scale, respectively. Throughout this article we follow the standard conventions and notations of the textbook of Misner, Thorne and Wheeler [11]. Note one important distinction between our model (1) and the Randall and Sundrum model [3, 4]. In the Randall and Sundrum model [3, 4] the geometry of the fifth dimension is taken to a singular orbifold geometry $S^{1} / Z_{2}$ [5], 6], whereas in our model the topology of the fifth

dimension is a circle $S^{1}$ because the existence of many domain wall solution for the Einstein equation requires us to choose this smooth manifold.

Variation of the action (四) with respect to the five-dimensional metric tensor leads to the Einstein equation:

$$
\sqrt{-g}\left(R^{M N}-\frac{1}{2} g^{M N} R\right)=-\sqrt{-g} g^{M N} \Lambda+\kappa^{2} \sum_{i=1}^{n} \sqrt{-g_{i}} g_{i}^{\mu \nu} \delta_{\mu}^{M} \delta_{\nu}^{N} \mathcal{L}_{i} \delta\left(z-L_{i}\right),
$$

where $M, N, \ldots$ denote five-dimensional indices, whereas $\mu, \nu, \ldots$ do four-dimensional ones. Provided that we adopt a metric ansatz

$$
\begin{aligned}
d s^{2} & =g_{M N} d x^{M} d x^{N} \\
& =u(z)^{2} \eta_{\mu \nu} d x^{\mu} d x^{\nu}+d z^{2},
\end{aligned}
$$

with $\eta_{\mu \nu}$ denoting the four-dimensional Minkowski metric, the Einstein equation (2) reduces to two combined differential equations for the unknown function $u(z)$ :

$$
\left(\frac{u^{\prime}}{u}\right)^{2}=k^{2}
$$




$$
\frac{u^{\prime \prime}}{u}=k^{2}+\frac{\kappa^{2}}{3} \sum_{i=1}^{n} \mathcal{L}_{i} \delta\left(z-L_{i}\right),
$$

where the prime denotes a differentiation with respect to $z$ and we have defined $k$ as

$$
k=\sqrt{-\frac{\Lambda}{6}}
$$

In the previous paper [8], we have seeked special solutions with the form of

$$
u(z)=e^{-k f(z)}
$$

and we have presented two distinct solutions with simple and manageable form although the other complicated solutions could be also constructed. These solutions describe even domain walls standing along $S^{1}$ at some intervals in five-dimensional anti-de Sitter space-time. One solution describes $\frac{n-1}{2}$ even domain walls locating at $L_{2 i}$ and is concretely given by

$$
\begin{aligned}
f(z) & =|z|+\sum_{i=1}^{\frac{n-1}{2}}(-1)^{i}\left|z-L_{2 i}\right|-L, \\
f^{\prime}(z) & =\sum_{i=1}^{\frac{n-1}{2}}(-1)^{i} \varepsilon\left(z-L_{2 i}\right)+1, \\
f^{\prime \prime}(z) & =2 \sum_{i=1}^{\frac{n-1}{2}}(-1)^{i} \delta\left(z-L_{2 i}\right)
\end{aligned}
$$

for which $\mathcal{L}_{i}\left(i=1,2, \cdots, \frac{n-1}{2}\right)$ must satisfy the relations

$$
\mathcal{L}_{2 i}=(-1)^{i+1} \frac{6 k}{\kappa^{2}}
$$

The other solution describes $n-1$ even domain walls locating at $L_{i}(i=1,2, \cdots, n-1)$ and takes the form

$$
\begin{aligned}
f(z) & =\sum_{i=2}^{n-1}(-1)^{i+1}\left|z-L_{i}\right|+L \\
f^{\prime}(z) & =\sum_{i=1}^{n-1}(-1)^{i+1} \varepsilon\left(z-L_{i}\right)-1 \\
f^{\prime \prime}(z) & =2 \sum_{i=1}^{n-1}(-1)^{i+1} \delta\left(z-L_{i}\right)
\end{aligned}
$$

for which, this time, $\mathcal{L}_{i}\left(i=1,2, \cdots, \frac{n-1}{2}\right)$ must satisfy the relations

$$
\mathcal{L}_{2 i}=-\mathcal{L}_{2 i-1}=\frac{6 k}{\kappa^{2}} .
$$


Moreover, in the both solutions, $L_{i}$ satisfies the relations

$$
L_{2 i}=\frac{L_{2 i-1}+L_{2 i+1}}{2}, L_{1} \equiv 0, L_{n} \equiv 2 L
$$

with $i=1,2, \cdots, \frac{n-1}{2}$.

To close this section, it is worthwhile to argue why it is difficult to construct a model consisting of only D3-branes with positive tension. This problem is indeed closely related to various important problems associated with the models under consideration. Before doing that, let us recall that in the original Randall-Sundrum model [3, 4], there are two 3-branes with opposite sign of brane tension at the boundaries of an orbifold $S^{1} / Z_{2}$. Also in our model, there are the same number of 3-branes with positive tension and negative tension on a circle $S^{1}$. It was stressed in Ref. [7] that the necessity for the negative energy objects might be one of disadvantages in those setups since not only it is believed that the Standard Model is placed on the D3-brane, which is certainly a positive energy object, but also some problematic facts were pointed out in the cosmological context. For instance, the Friedmann-like expanding universe does not arise if the Standard Model is placed on the 3-brane with negative brane tension [12, 13]. Actually, in Ref.[3], the Standard Model was located on such a negative energy 3-brane in order to explain the exponential mass hierachy.

Keeping these facts in mind, let us now turn to our question: "Why is it difficult to construct a model consisting of only many D3-branes with positive tension?" The answer can be easily found from considerations of the electro-maganetics in a compact space. It is well known that we cannot put a single point charge in a compact space since electric flux lines have no place to go in the compact space. In other words, the field equation does not admit the existence of such a configuration. To remedy it, the simplest way is to introduce another point charge with the same size but opposite sign to the first point charge in order to make the flux lines exactly close. The other simplest way is to take account of a non-compact space by taking the limit of infinitely large size. In a non-compact space, the flux lines arising from a single point charge could run to infinity so we could have a consistent model with any number and configuration of 3-branes. In fact, this latter procedure has been recently taken by Lykken and Randall [7] at least implicitly in order to make a model with only positive tension 3-branes.

Incidentally, it is of interest to observe that a similar situation also occurs in the context of D-brane theory [14]. The D-brane, unlike the fundamental string, carries positive R-R charge. We cannot therefore put a single (or many) D-brane(s) in a compact region owing to a non-zero total $\mathrm{R}-\mathrm{R}$ charge. To cancel the $\mathrm{R}-\mathrm{R}$ charge exactly, we are led to introduce some objects which carry negative $\mathrm{R}-\mathrm{R}$ charge with the same size. They are nothing but orientifolds! From this analogy, it is interesting to regard 3-branes with positive and negative tension as D3-branes and O3-planes, respectively. We think that we should pursue this analogy further to understand a long-standing problem, i.e., the cosmological constant problem, in future. 


\section{A model with positive tension D3-branes}

In this section, on the basis of the solutions given in the previous section, we will present a concrete model which realizes simultaneously mass hierarchy and trapping of gravity on our universe ("visible 3-brane"). An essential aspect with respect to the two phenomena is very similar in the two distinct solutions Eqs.(8), (10), so we limit ourselves to the type of the solution Eq.(10) in this paper. Moreover, in this section and the next section, to start with, we shall give a model in the case of two positive tension 3-branes and then extend it to a more general case of arbitrary number of positive tension 3-branes.

Let us start with a model with two positive tension branes and two negative tension branes, which is a specific example $(n=5)$ of the solution (10):

$$
\begin{aligned}
f(z) & =-\left|z-L_{2}\right|+\left|z-L_{3}\right|-\left|z-L_{4}\right|+L \\
f^{\prime}(z) & =\varepsilon(z)-\varepsilon\left(z-L_{2}\right)+\varepsilon\left(z-L_{3}\right)-\varepsilon\left(z-L_{4}\right)-1 \\
f^{\prime \prime}(z) & =2\left[\delta(z)-\delta\left(z-L_{2}\right)+\delta\left(z-L_{3}\right)-\delta\left(z-L_{4}\right)\right] .
\end{aligned}
$$

Of course, $\mathcal{L}_{i}$ must satisfy the relation (11) specified to $n=5$ case:

$$
\mathcal{L}_{1}=-\mathcal{L}_{2}=\mathcal{L}_{3}=-\mathcal{L}_{4}=-\frac{6 k}{\kappa^{2}}
$$

Here, instead of Eq.(12) we require slightly modified relations for $L_{i}$ :

$$
L=L_{2}-L_{3}+L_{4}, L_{1} \equiv 0, L_{n} \equiv 2 L, L_{3}>2 L_{2} .
$$

The reason is that as mentioned in the paper [8], the solution (10) with the relation (12) has a characteristic feature $f\left(L_{2 i-1}\right)=0$ (or equivalently, $u\left(L_{2 i-1}\right)=1$ ) which is a undesired feature in explaining mass hierarchy. Thus, in order to avoid this feature, we have imposed the relation (15), specially, $L_{3}>2 L_{2}$ on the solution (13). Indeed, as shown in the next section, this type solution has a desired feature with respect to both mass hierarchy and trapping of gravity. And note that the positive tension domain walls are located at $z=L_{1} \equiv 0, L_{3}$ while the negative tension domain walls are at $z=L_{2}, L_{4}$ as seen in Eq.(14). For comparison with Ref. [7], according to their terminology, we may call the positive tension 3-branes at $z=L_{1} \equiv 0$ and at $z=L_{3}$ "TeV brane" and "Planck brane", respectively fl. It might appear to be difficult to move only the two negative tension branes to infinity without changing the essential contents of the model since the negaive and the positive tension branes are adjacent in the solution (13). But the analysis in the next section reveals that this procedure can be carried out by taking the limit of $L_{2}, 2 L-L_{4} \rightarrow \infty$ with keeping $L_{3}-2 L_{2}$ finite. In this way, we can construct a model consisting of only two positive tension domain walls separated at some distance along a non-compact fifth dimension.

\footnotetext{
${ }^{2}$ Our setup is different from that of Ref.[7] where "Planck brane" and "TeV brane" are put at $z=L_{1} \equiv 0$ and at $z=L_{3}$, respectively. One can modify the present setup to coincide with their setup without any difficulty.
} 
Next let us present a model including general $\frac{n-1}{2}$ positive tension domain walls which is a straightforward generalization of the model with two positive tension domain walls. This model is just given in terms of Eq.(10) satisfying Eq.(11) but modified relations among $L_{i}$ compared to Eq.(12), and a limiting procedure. For convenience, let us write down explicitly this general model with $\frac{n-1}{2}$ positive tension D3-branes:

$$
\begin{aligned}
f(z) & =\sum_{i=2}^{n-1}(-1)^{i+1}\left|z-L_{i}\right|+L, \\
f^{\prime}(z) & =\sum_{i=1}^{n-1}(-1)^{i+1} \varepsilon\left(z-L_{i}\right)-1, \\
f^{\prime \prime}(z) & =2 \sum_{i=1}^{n-1}(-1)^{i+1} \delta\left(z-L_{i}\right), \\
\mathcal{L}_{2 i} & =-\mathcal{L}_{2 i-1}=\frac{6 k}{\kappa^{2}}, \\
L & =\sum_{i=2}^{n-1}(-1)^{i} L_{i}, L_{1} \equiv 0, L_{n} \equiv 2 L,
\end{aligned}
$$

with $i=1,2, \cdots, \frac{n-1}{2}$. And the limiting procedure is given by

$$
f\left(L_{2 i}\right) \rightarrow \infty
$$

with keeping $f\left(L_{2 i-1}\right)$ some finite, negative values.

\section{Exponential mass hierarchy and Newton's law}

In the previous section, we have presented a concrete model so we are now ready to consider how this model resolves problems of mass hierarchy and trapping of gravity. As in the previous section, let us start with the model with two positive tension domain walls. Following the formula given in Ref. [8], it is straightforward to evaluate mass scale $m(0)$ on the "TeV brane" located at $z=L_{1} \equiv 0$ from mass scale $m\left(L_{3}\right)$ on the "Planck brane" located at $z=L_{3}$ to which the Planck mass scale is allocated. The result is of the form

$$
m(0)=e^{-k\left(L_{3}-2 L_{2}\right)} m\left(L_{3}\right) .
$$

As stated in the previous section, since we have taken $L_{3}-2 L_{2}$ to be a positive and finite value, we can resolve the mass hierarchy if $L_{3}-2 L_{2}$ is of order of 10 . In other words, the mass scale in our universe ("TeV brane") is of order electroweak scale thanks to the red-shift factor in the geometry when the Planck mass scale is assigned to another positive tension domain wall ("Planck brane") at $z=L_{3}$ and $L_{3}-2 L_{2}$ is of order of 10 .

Next, let us turn our attention to a problem of trapping of gravity on "TeV brane". To so that, we will consider the linearlized approximation of the metric tensor and examine small 
fluctuations $h_{\mu \nu}$ around the four-dimensional Minkowski metric on the brane and determine the graviton spectrum as well as the Kaluza-Klein spectrum. Accordingly, we assume the form

$$
\begin{aligned}
d s^{2} & =g_{M N} d x^{M} d x^{N} \\
& =e^{-2 k f(z)}\left(\eta_{\mu \nu}+h_{\mu \nu}(x, z)\right) d x^{\mu} d x^{\nu}+d z^{2} .
\end{aligned}
$$

Then, with the gauge conditions $h_{\mu}^{\mu}=\partial^{\mu} h_{\mu \nu}=0$, up to the leading order of $h_{\mu \nu}$, the field equation is of the form

$$
-\frac{1}{2} e^{2 k f(z)} \square h_{\mu \nu}-\frac{1}{2} h_{\mu \nu}^{\prime \prime}+2 k^{2} h_{\mu \nu}-k f^{\prime \prime}(z) h_{\mu \nu}=0,
$$

where $\square$ denotes the flat space-time four-dimensional Laplacian operator, and $f(z)$ is defined as in Eq.(13). At this stage, if we consider the plane wave fluctuations

$$
h_{\mu \nu}(x, z)=e^{i p_{\mu} x^{\mu}} h_{\mu \nu}(z), p_{\mu}^{2}=-m^{2},
$$

Eq.(20) reduces to the differential equation for $h_{\mu \nu}(z)$

$$
\left[-\frac{m^{2}}{2} e^{2 k f(z)}-\frac{1}{2} \partial_{z}^{2}+2 k^{2}-k f^{\prime \prime}(z)\right] h_{\mu \nu}(z)=0,
$$

It is more convenient to rewrite Eq.(22) into the one-dimensional Schrodinger waveequation by making a change of variables, $y=\frac{1}{k} e^{k f(z)}$

$$
\left[-\frac{1}{2} \partial_{y}^{2}+V(y)\right] \Psi(y)=\frac{m^{2}}{2} \Psi(y),
$$

where we have defined as

$$
h_{\mu \nu}(z) \equiv k^{-\frac{1}{2}} y^{-\frac{1}{2}} \Psi_{\mu \nu}(y), \Psi_{\mu \nu}(y) \equiv \Psi(y) .
$$

The one-dimensional potential is now given by

$$
V(y)=\frac{15}{8} \frac{1}{y^{2}}-\frac{2}{y}\left[\delta\left(y-y_{1}\right)-\delta\left(y-y_{2}\right)+\delta\left(y-y_{3}\right)-\delta\left(y-y_{4}\right)\right],
$$

where $y_{i}(i=1,2,3,4)$ are of the form

$$
\begin{aligned}
y_{1} & =\frac{1}{k} \\
y_{2} & =\frac{1}{k} e^{k L_{2}}, \\
y_{3} & =\frac{1}{k} e^{-k\left(L_{3}-2 L_{2}\right)}, \\
y_{4} & =\frac{1}{k} e^{k\left(2 L-L_{4}\right)},
\end{aligned}
$$


thus we have a relation

$$
0<y_{3}<y_{1}<y_{2}<y_{4}
$$

where without loss of generality we have assumed $y_{2}<y_{4}$. Here an interesting thing has happened owing to Eq.(15). Namely, the change of variables from $z$ to $y$ has caused a rearrangement of the positions of domain walls where the two positive tension 3-branes are located at the left side of the two negative tension 3-branes, so we can move the two negative tension 3-branes to infinity by taking the limit of $L_{2}, 2 L-L_{4} \rightarrow \infty$ with keeping $L_{3}-2 L_{2}$ finite. Note that the two positive tension 3-branes stay at the same points in this limit.

The one-dimensional Schrodinger wave-equation Eq.(23) gives rise to much useful informations about the graviton and a tower of the Kaluza-Klein modes. Luckily enough, the potential $V(y)$ has a similar form to that in Refs. [4, 7] so that we can take over the results obtained there to the present case with an appropriate modification. First of all, the potential $V(y)$ has the $\delta$-functions at $y=y_{1}, y_{3}$ with negative coefficients, which means that these $\delta$-functions supports a normalizable bound state mode, which is of course nothing but the massless graviton. Incidentally, the "regulator" branes with negative brane tension locating at $y=y_{2}, y_{4}$, though they are moved to infinity, induce the $\delta$-functions with positive coefficients into the potential $V(y)$, so these branes cannot support such a massless graviton. This is the reason that in Ref. [4], Randall and Sundrum have regarded the 3-brane with positive tension as our universe for the trapping of graviton on the brane.

Second, let us focus our attention to the properties of the continuous KK modes. In this point, in the leading approximation, we can safely neglect the existence of the $\delta$-functions in the potential $V(y)$. Then, it turns out that the squared mass $m^{2}$ of the KK modes is positive definite as desired. In the non-compact limit, the potential falls off to zero, so there is no mass gap between the massless graviton and the KK modes. At first sight, this could be a signal of danger since a tower of KK modes would give measurable effects to the modification of the Newton's law, but as shown shortly, they give rise to only small corrections to the Newton's law [4, 7]. We can easily write down the general solution for the continuous KK modes as

$$
\Psi_{m}(y) \sim \frac{m^{\frac{5}{2}}}{k^{2}} \sqrt{y}\left[Y_{2}(m y)+\frac{4 k^{2}}{\pi m^{2}} J_{2}(m y)\right],
$$

where $Y_{2}$ and $J_{2}$ denote the Bessel functions of order 2. From the above wave function, it is straightforward to evaluate the corrections to the Newton's law from the continuous KK modes [4, 7]. In fact, the gravitational potential between two masses $m_{1}$ and $m_{2}$ takes the form in the polar coordinate

$$
U(r)=G_{N} \frac{m_{1} m_{2}}{r}+\int_{0}^{\infty} \frac{d m}{k} G_{N} \frac{m_{1} m_{2} e^{-m r}}{r}=G_{N} \frac{m_{1} m_{2}}{r}\left(1+\frac{1}{k^{2} r^{2}}\right) .
$$

Accordingly, it turns out that an observer living in the "TeV brane" (and also "Planck brane") sees gravity as essentially four-dimensional since the corrections to the Newton's law from the KK modes are very small. (Note that $k$ is taken to be of order the Planck scale.) 
In the above, the case of the two D3-branes with positive tension has been investigated in detail. The analysis of the general model Eqs.(16) and (17) is quite straightforward and very similar to the case of the two D3-branes, so that we will not repeat it in this paper. For instance, in the Schrodinger wave-equation (23), only the modification lies in the form of the potential $V(y)$ where $n-1 \delta$-functions appear instead of $4 \delta$-functions. Hence, we can show the trapping of gravity on the D3-branes again. On the other hand, for the hierarchy problem, one can also show that the electroweak scale is generated on our universe when the Planck scale is assigned to a hidden universe and an appropriate value of distance between the two universes is chosen. However, in this general model, this is not the whole story since there are many red-shift factors arising from relative distances between our universe and the remaining hidden universes. This issue is worthy of further study and will be reported in detail in a separate publication [10].

\section{Discussions}

In this paper, we have investigated problems of both mass hierarchy and trapping of gravity on our universe by using the solutions describing many domain walls which were previously found by the present author [8]. Our model in general consists of any number of positive tension D3-branes in a non-compact extra dimension, and shows the exponential mass hierarchy and trapping of gravity in our universe. In this sense, the study at hand shows that the scenario by Lykken and Randall [7] is indeed realized.

Since various matters and gauge fields are also localized on our D3-brane universe in terms of the mechanism in string theory, our model is equipped with necessary conditions as a realistic model. Thus, future directions of this work would be to apply our model to other unsolved problems. In fact, we can easily point out that our model has the following advantages over the original Randall-Sundrum model. In the context of cosmology, since there exist only positive tension D3-branes in our model, the lack of the Friedmann-like expanding universe is certainly resolved [12, 13]. And, in the context of phenomenology, some of puzzles stated in a recent study [9] seem to be also resolved in terms of our general model including many D3-branes since we have not a single but many "red-shift" factors associated with many domain walls. These problems will be reported in a separate publication [10.

\section{References}

[1] N. Arkani-Hamed, S. Dimopoulos and G. Dvali, Phys.Lett.B429 (1998) 263, hepth/9803315. 
[2] I. Antoniadis, N. Arkani-Hamed, S. Dimopoulos and G. Dvali, Phys.Lett.B436 (1998) 257 , hep-th/9804398.

[3] L. Randall and R. Sundrum, hep-ph/9905221.

[4] L. Randall and R. Sundrum, hep-th/9906064.

[5] P. Horava and E. Witten, Nucl.Phys.B460 (1996) 506, hep-th/9510209; E. Witten, Nucl.Phys.B471 (1996) 135, hep-th/9602070; P. Horava and E. Witten, Nucl.Phys.B475 (1996) 94 , hep-th/9603142.

[6] A. Lukas, B.A. Ovrut, K.S. Stelle and D. Waldram, hep-ph/9803235.

[7] J. Lykken and L. Randal, hep-th/9908076.

[8] I. Oda, hep-th/9908104.

[9] K.R. Dienes, E. Dudas and T. Gherghetta, hep-ph/9908530.

[10] I. Oda, to appear.

[11] C.W. Misner, K.S. Thorne and J.A. Wheeler, Gravitation, San Francisco: Freeman.

[12] C. Csaki, M. Graesser, C. Kolda and J. Terning, hep-ph/9906513.

[13] J. Cline, C. Grojean and G. Servant, hep-ph/9906523.

[14] J. Polchinski, "Tasi Lectures on D-branes", hep-th/9611050. 